\title{
Genetic Research and Women's Heart Disease: a Primer
}

\author{
Maryam Kavousi $^{1}$ - Lawrence F. Bielak ${ }^{2}$ - Patricia A. Peyser ${ }^{2}$
}

Published online: 10 October 2016

(C) The Author(s) 2016. This article is published with open access at Springerlink.com

\begin{abstract}
Purpose of Review This review provides a brief synopsis of sexual dimorphism in atherosclerosis with an emphasis on genetic studies aimed to better understand the atherosclerotic process and clinical outcomes in women. Such studies are warranted because development of atherosclerosis, impact of several traditional risk factors, and burden of coronary heart disease (CHD) differ between women and men.

Recent Findings While most candidate gene studies pool women and men and adjust for sex, some sex-specific studies provide evidence of association between candidate genes and prevalent and incident CHD in women. So far, most genomewide association studies (GWAS) also failed to consider sexspecific associations. The few GWAS focused on women tended to have small sample sizes and insufficient power to reject the null hypothesis of no association even if associations exist.

Summary Few studies consider that sex can modify the effect of gene variants on CHD. Sufficiently large-scale genetic studies in women of different race/ethnic groups, taking into account possible gene-gene and gene-environment interactions as well as hormone-mediated epigenetic mechanisms, are needed. Using the same disease definition for women
\end{abstract}

This article is part of the Topical Collection on Women and Ischemic Heart Disease

Maryam Kavousi

m.kavousi@erasmusmc.nl

1 Department of Epidemiology, Erasmus University Medical Center, Rotterdam, P.O. Box 2040, 3000 CA Rotterdam, The Netherlands

2 Department of Epidemiology, School of Public Health, University of Michigan, 1415 Washington Heights, Ann Arbor, MI 48109-2029, USA and men might not be appropriate. Accurate phenotyping and inclusion of relevant outcomes in women, together with targeting the entire spectrum of atherosclerosis, could help address the contribution of genes to sexual dimorphism in atherosclerosis. Discovered genetic loci should be taken forward for replication and functional studies to elucidate the plausible underlying biological mechanisms. A better understanding of the etiology of atherosclerosis in women would facilitate future prevention efforts and interventions.

Keywords Cardiovascular disease $\cdot$ Coronary heart disease . Atherosclerosis $\cdot$ Women $\cdot$ Genes $\cdot$ Sex-differences

\section{Introduction}

Cardiovascular disease (CVD) remains the leading cause of mortality among women and men [1,2]. Although overall CVD mortality rates have declined, the annual mortality rate for women remains higher than men [3]. Greater life expectancy for women, together with improvements in primary and secondary prevention of CVD, will lead to a larger proportion of women living with CVD [3]. Substantial sex differences in the burden of different manifestations of CVD, including coronary heart disease (CHD), stroke, heart failure, and peripheral artery disease, are widely recognized [4-7]. Despite the excess CHD incidence and prevalence in men compared to women, CHD remains the leading contributor to CVD morbidity and mortality among both women and men $[1,2]$.

In spite of statistics that show CHD develops on average 710 years later in women compared with men, adverse trends in many risk factors among women are of growing concern [3, 8]. Additionally, while the decrease in CHD mortality among women is well documented, the decline still lags behind that of men, with an alarming tendency towards an increase 
mortality rate among younger women $[9,10 \bullet]$. Recent data report substantial declines in sudden cardiac death in men while no changes are observed among women [11].

We provide a review of sex differences in atherosclerosis, the contribution of genetic research to explaining sex differences in atherosclerosis, possible sex hormone-mediated epigenetic mechanisms, and use of subclinical measures of atherosclerosis for genetic studies. Next, we discuss challenges in accounting for sex differences in genetic studies, importance of the proper definition of outcomes, the need to include multiple race/ethnic groups in genetic studies of atherosclerosis in women, and how this genetic information can contribute to efforts in precision medicine for CHD in women. We conclude with directions for future research, limitations of this review, and conclusions. Here, sex refers to biological differences between women and men (i.e., anatomical and physiological differences, genetic differences in the $\mathrm{X}$ and $\mathrm{Y}$ chromosomes, and levels and types of hormones). Gender is "the socially constructed characteristics of women and men - such as norms, roles and relationships of and between groups of women and men. It varies from society to society and can be changed" (http://www.who.int/gender-equityrights/understanding/gender-definition/en/).

\section{Sex Differences in Atherosclerosis}

CHD is mainly characterized by atherosclerosis in the epicardial coronary arteries. Atherosclerosis is a systemic progressive pathologic condition involving atherosclerotic plaque formation typified by accumulation of cholesterol, infiltration of macrophages, proliferation of smooth muscle cells, accumulation of connective tissue components, and formation of thrombus [12]. Atherosclerosis is considered a complex trait involving multiple genes and their interactions with behavioral and environmental factors. Complex traits do not follow predictable patterns of inheritance. Although women and men share many similarities in core processes underlying atherosclerosis, recent evidence points towards some inherent differences in the development of the disease. Sex differences in the association of traditional risk factors with $\mathrm{CHD}$ are established. In particular, smoking and diabetes seem to be stronger risk factors in women $[13,14]$. Sex differences, however, cannot be entirely explained by the differential distribution of traditional risk factors between women and men. Furthermore, women tend to show a more "diffuse atherosclerosis" pattern, as opposed to discrete "focal atherosclerosis" that obstructs the lumen [15].Compared to men, women tend to have a higher prevalence of microvascular dysfunction [15, 16]. Plaque characteristics relative to calcification and lipid accumulation may also differ between women and men, and transitions toward vulnerable plaques seem to be slower in females [15]. Sex differences in inflammatory, coagulation, and thrombotic pathways may contribute to this sexual dimorphism [15-17]. The exact pathways associated with atherosclerosis in women and men, however, remain to be elucidated.

\section{Contribution of Genetic Research to Explaining Sex Differences in Atherosclerosis}

The heritability of CHD is approximately $40 \%$ [18]. Several studies have shown that a family history of CHD, especially when disease occurs before age 60 years, is more important for women than men [19]. Understanding the genetic basis of sexual dimorphism in atherogenesis may provide novel additions to existing knowledge.

\section{Candidate Genes for Atherosclerosis}

A gene whose function or location indicates it is likely to be responsible for a particular disease or trait level is referred to as a candidate gene. Detailed information about specific genes can be found at http://www.ncbi.nlm.nih.gov/gene. Most candidate gene studies pool women and men and use sex as a covariate for adjustment. In the selected studies described below, men and women were considered separately. While some studies found associations in men, but not women, we only report those where an association only occurred in women.

\section{Apolipoprotein E}

One of the most studied candidate genes for CHD is apolipoprotein E (APOE) [20]. APOE produces a protein involved in metabolism of cholesterol and triglycerides by binding to receptors in the liver to promote clearance of chylomicrons and very low-density lipoproteins from circulating blood [20]. The major alleles of APOE are $\varepsilon 2, \varepsilon 3$, and $\varepsilon 4$. The most common genotypes are $\varepsilon 3$ homozygotes, $\varepsilon 3 / \varepsilon 4$ heterozygotes, and $\varepsilon 2 /$ $\varepsilon 3$ heterozygotes in most populations. $\varepsilon 2 / \varepsilon 3$ heterozygotes have higher high-density lipoprotein cholesterol (HDL-C) levels while $\varepsilon 3 / \varepsilon 4$ heterozygotes have higher low-density lipoprotein cholesterol (LDL-C) levels compared to $\varepsilon 3$ homozygotes [20]. $\varepsilon 3 / \varepsilon 4$ heterozygotes have higher risk of CHD compared to $\varepsilon 3$ homozygotes and $\varepsilon 2 / \varepsilon 3$ heterozygotes, while there is no significant difference in CHD risk between $\varepsilon 3$ homozygotes and $\varepsilon 2 / \varepsilon 3$ heterozygotes [20].

The Framingham Offspring Study, one of the earliest studies to demonstrate a gender-specific association between $\varepsilon 4$ and prevalent CHD, found a significant positive association in women, but not men, after adjusting for age and traditional CHD risk factors [21]. No protective effect of $\varepsilon 2$ was found in either women or men in this study of 1034 men and 916 women. More recently, a large prospective study of 10,035 men and 12,134 women from the Norfolk, England, arm of the European Prospective Investigation into Cancer and Nutrition study (EPIC Norfolk study), reported no association 
between CHD risk and $A P O E$ in either men or women after adjustment for multiple traditional risk factors [22]. While both the Framingham Study and the EPIC Norfolk study adjusted for LDL-C and HDL-C levels, the Framingham Study assessed prevalence of CHD while the EPIC Norfolk study assessed incident CHD over 11 years. Finally, the EPIC Norfolk study also included alcohol use and physical activity as risk factors. The large, prospective EPIC Norfolk study supports a limited role for $A P O E$ in risk of CHD for either sex after accounting for many CHD risk factors [22]. Importantly, these studies suggest that genes for prevalent CHD may differ from genes for incident CHD.

\section{Selected Other Candidate Genes}

In two large Finnish cohorts, 46 candidate genes were studied for association with CHD [23]. No variants in any candidate gene were associated with incident CHD in men. Variants in three genes, however, showed an association with incident CHD in women: upstream stimulatory factor 1 (UFS1), coagulation factor XIII A (F13A1), and carboxypeptidase B2 (CPB2). UFS1, which was also associated with prevalent $\mathrm{CHD}$ in women, is a ubiquitously expressed transcription factor that regulates several genes of glucose and lipid metabolism. F13A1 is involved in the blood coagulation cascade while $C P B 2$ is involved in fibrinolysis. Additionally, mutations in either of two polymorphisms in the hemochromatosis (HFE) gene in the Rotterdam Study were significantly associated with incident CHD in women, but not men [24]. HFE regulates circulatory iron uptake. All the candidate gene studies described above included only individuals of European ancestry.

The candidate gene approach has been criticized primarily because many results could not be replicated and this approach fails to include all possible causative genes and polymorphisms [25]. These criticisms spurred new approaches such as genome-wide association studies (GWAS) to identify genes for complex diseases and traits

\section{Genome-Wide Association Studies}

GWAS typically consider millions of single-nucleotide polymorphisms (SNPs) (or other genetic variants such as copy number polymorphisms). Genetic variants are measured with microarrays, and the measures from the microarrays are often combined with publically available data, such as the 1000 Genomes Project, to impute additional genotypes that were not directly measured. The 1000 Genomes Project has provided genomic sequence data on more than 2500 individuals from 26 globally diverse populations [26].

In contrast to candidate gene studies, there are no specific a priori hypotheses in GWAS with the exception of replication of already identified genes. These studies typically meta- analyze results from multiple cohorts to increase power. There is usually a discovery phase that includes the initial cohorts and then a replication phase with additional new cohorts. Cohorts from the discovery and replication phases may be meta-analyzed together. Depending on the number of SNPs considered, the $p$ value is set to meet a Bonferroni correction. Many GWAS of traditional CHD risk factors explore whether any identified genes are also associated with clinical outcomes. A catalogue, continually updated, of GWAS findings is available $[27 \cdot \bullet]$.

The first GWAS papers on CHD were published in 2007 [28-32]. The studies pooled women and men of European ancestry and adjusted for sex as a covariate. The most consistent finding in these studies was an association with variants on the short arm of chromosome 9 (9p21).

Recent GWAS have included more genetic variants and larger sample sizes. The most recent GWAS included 60,801 CHD cases and 123,504 controls from 48 different cohorts [33••]. While most of the cases and controls were of European ancestry, some were of other ancestries. The study interrogated 9.4 million variants across the genome. Ten new loci were identified bringing the total number of loci associated with CHD to $58[33 \bullet \bullet, 34 \bullet \cdot]$.

Sex-specific associations for CHD were conducted in the Wellcome Trust Case Control Consortium. In women, there were 399 cases for $\mathrm{CHD}$ and 1492 controls while in men there were 1527 cases and 1446 controls. Variants considered were selected from results of other GWAS for CHD. No variants were significant in women (or in men) [35]. Power was low in this study.

In a recent sex-stratified study, an SNP in SCARB1, a plasma membrane receptor for HDL, was associated with angiographic CHD in women, but not men [36•]. A GWAS of betaine levels, a novel risk factor for atherosclerosis, found an association with variation in a SNP in carbamoylphosphate synthase 1 (CPS1) [37••]. CPS1 encodes a mitochondrial enzyme that catalyzes the first committed reaction and rate-limiting step in the urea cycle. This SNP was weakly associated $(p=0.01)$ with CHD in approximately 54,000 individuals from the CARDIoGRAM Consortium [38]. The SNP was not associated with CHD in men; however, it was significantly associated with CHD in women [37••].

All CHD GWAS so far focused exclusively on the autosomes even though the $\mathrm{X}$ chromosome is included on all the current microarrays [39]. The X chromosome contains 1973 known genes. Even with the challenges of including the $\mathrm{X}$ chromosome in GWAS because women have two X chromosomes while men have one and because of X-inactivation, this chromosome may provide important information regarding differences in atherosclerosis and its risk factors between women and men. The one exception is a recent GWAS of nonobstructive coronary artery disease in women that included 52,371 variants important in metabolic traits and CVD [40, 
41]. Ninety variants were on the $X$ chromosome. The 332 European ancestry cases came from a cohort of women with chest pain and/or suspected myocardial ischemia with $<50 \%$ stenosis in any coronary artery on angiography. The 1003 European ancestry controls came from a cohort of women without known CHD. While there were no associations with any SNPs on the X chromosome, SNPs at two autosomal genes showed association at nominal significance levels. In a candidate gene study of $5 H T R 2 C$ on the $\mathrm{X}$ chromosome, men who had one copy of the high-risk allele and women with two copies of the high-risk allele were at significantly increased risk for death or nonfatal myocardial infarction [42].

\section{Sex Hormones and Epigenetics}

CHD in women tends to manifest during and after the menopausal transition, indicating that sex hormones play a critical role in disease development. The major impact of sex hormones on atherosclerosis, either directly affecting the function of the heart and vessels or indirectly through other CHD risk factors, has long been investigated [43]. A recent understanding of the interaction of genes with the environment has revealed the importance of sex hormones on pathogenesis of atherosclerosis through epigenetic mechanisms.

Epigenetics refers to heritable changes in gene activity and expression that do not entail an alteration in DNA sequence. In other words, epigenetics involves the molecular pathways that modulate the expression of a genotype into a particular phenotype [44]. Epigenetic modifications often investigated include DNA methylation, histone variants, and histone modifications as well as nucleosome positioning [45]. Epigenetic mechanisms are reversible and can be modulated by environmental factors [46]. Thus, epigenetics has emerged as a promising tool to address knowledge gaps in atherosclerosis.

CHD risk factors, such as nutrition, smoking, pollution, stress, and the circadian rhythm, have been associated with epigenetic modifications [47•]. Additionally, sex hormones are uniquely poised to exert epigenetic effects through hormone-induced DNA methylation and histone modification at specific gene regulatory regions [48]. Any evidence regarding a sex-specific association of a particular genetic locus with atherosclerosis could indicate underlying epigenetic mechanisms mediated through sex hormones.

\section{Subclinical Measures of Atherosclerosis}

The distribution of atherosclerosis in different vascular beds is variable and sex- and race/ethnicity-specific [49-52]. Differences in atherosclerosis between men and women have been suggested to be larger in the coronary arteries than in other vascular beds [49]. Calcification in the coronary arteries is a surrogate marker of overall plaque burden and is considered the hallmark of atherosclerosis. While women have overall lower presence and quantity of coronary artery calcification (CAC) than men, CAC carries a higher mortality risk for women [53]. The quantity of CAC is heritable [54]. So far, none of the GWAS for $\mathrm{CAC}$ have considered men and women separately $[55 \bullet, 56]$. The GWAS for CAC in those of European ancestry identified associations with SNPs in the 9p21 region and in PHACTR1 on chromosome 6 that replicated for myocardial infarction [55•]. A GWAS for CAC in those of African ancestry failed to identify any genome-wide significant associations [56].

Few candidate gene studies have evaluated genetic associations for CAC in men and women separately. A polymorphism in E-selectin, a gene involved in cellular adhesion, was associated with presence of CAC only in women age 50 years or younger after adjustment for $\mathrm{CHD}$ risk factors. There was no association with CAC presence in men of any age or in women over age 50 after adjustment for CHD risk factors [57]. A promoter polymorphism of leukotriene C4 synthase (LTC4S), the rate-limiting enzyme in the production of the potent proinflammatory cysteinyl leukotriene metabolites of arachidonic acid, was studied in women ages 29-43 years and men ages 2937 years [58]. Risk for having CAC was significantly associated with this polymorphism in women, but not men.

Increased common carotid intima-media thickness (CIMT) is considered a marker of early atherosclerosis. Sex differences in CIMT seem to be pertinent only in younger and middle-aged individuals, becoming progressively irrelevant at older age [59]. In a longitudinal study of CIMT, progression of CIMT was associated with parental history of stroke especially among young women [60]. CIMT is heritable [61]. A GWAS in those of European ancestry identified variants near ZHX2, ACPOC1, and PINX1 associated with CIMT and did not consider women and men separately [62•]. A recent GWAS in a study that included individuals from multiple race/ethnic groups identified 14 genes with evidence for an association with CIMT [63•]. An SNP-by-sex interaction was found for a SNP in LEKR1 and an SNP in GALNT10. Both of these loci have been associated with adiposity and weight control [63•]. Similar to the association with CAC described above, LTC $S$ was associated with CIMT in women but not in men [58]. Others have identified a locus on chromosome 16 that is associated with CIMT, which contains the BCAR1, CFDP1, and TMEM170A genes [64]. More recently, Boardman-Pretty and colleagues [65 ${ }^{\circ}$ studied the lead SNP in $B C A R 1$, identified its function, and showed it to be associated with progression of CIMT in women, but not men.

Endothelial function, a measure of physiological functions of the vascular endothelium, is a marker representing the effects of CHD risk factors on the arterial wall [66, 67]. Functional and structural damages to the arterial wall precede and accompany atherosclerosis process and its associated obstructive and thrombotic events [66]. Sex has been suggested as an independent factor contributing to endothelial 
dysfunction, and the effect of cardiovascular risk factors on endothelium-dependent dilation has been shown to be sexspecific [68, 69]. Endothelial function is heritable [70]. An early GWAS did not find any genome-wide significant associations but found some suggestive associations for endothelial function [71]. A recent study investigated the association between almost 1300 SNPs previously associated with vasoreactivity, angiogenesis, inflammation, artery calcification, atherosclerotic risk factors, insulin resistance, hormone levels, blood coagulability, or CHD with coronary endothelial dysfunction [72]. SNPs in LPA, MYBPH, ADORA3, and $P O N 1$ were significantly associated in the 426 women, but not in the 217 men.

\section{Accounting for Sex Differences in Genetic Studies: the Challenge}

Analyzing sex differences in genetic associations is complicated, particularly for a disease such as CHD where the typical age of onset is gender-dependent. Sufficiently powered studies are an essential requirement in both candidate gene and GWAS studies. Lack of adequate statistical power has hampered meaningful comparisons between women and men in most of the numerous published GWAS for CHD so far. The sex-specific GWAS in the Wellcome Trust Case Control Consortium [35] and the study of nonobstructive coronary disease in women [40] illustrate the challenges of smaller samples and the need for large-scale studies in women.

Importantly, when women and men are pooled in a study, the analysis of any variant, but especially rare genetic variants, might be prone to bias resulting from the disproportionate blend of women and men in case and control samples. Other genetic or environmental factors can modify or mediate the effect of a particular genetic variant to increase or decrease the risk for CHD in women. For example, a study with a large sample of men and women found an interaction between smoking and $A P O E$ on CHD risk [73]. Women, but not men, had an increased smoking-related risk in $\varepsilon 4$ allele carriers. Differences between men and women can result from the joint effects of genetic variants with other biological and/or environmental variables.

While investigating sex differences in genetic studies of atherosclerosis are of paramount importance, researchers should be aware not to overstate sex differences in their studies, as spurious claims of sex differences in genetic studies might be asserted in the absence of sufficient data, proper data analysis, or good internal and external validity [74•]. Bias against reporting negative or null results can lead to more publications conveying findings of sex differences compared to studies with no sex differences appearing in the literature [75].

Since the sex-specific differences may be larger for causal variants than for their linked markers that might be studied, systematic assessment should be aimed at targeting the true causal variants associated with atherosclerosis [35]. Candidate gene studies should be based on a priori, clearly defined hypotheses. Any claim of sex-specific genetic association should be accompanied by appropriate examination of subgroup comparisons or interaction tests. Likewise, gene-gene and gene-environment interactions should be interpreted with caution and viewed in the context of additional prior/external evidence. The identified interactions should be interpreted as hypothesis-generating, followed by further replication in other studies as well as meta-analyses $[76,77]$.

\section{Proper Definition of Outcome in Sex-specific Genetic Studies of Atherosclerosis}

In studies of complex disease, defining a trait with insufficient specificity, i.e., trait heterogeneity, is viewed as a confounding factor [78]. The pathophysiology of CHD involves more severe structural and functional abnormalities in epicardial coronary arteries in men and more microvascular coronary dysfunction in women [79]. Thus, using the same definition for the CHD phenotype for both women and men might not be appropriate and could lead to differential misclassification of cases and controls between women and men. For example, in the most recent GWAS for CHD, case status was defined by an inclusive $\mathrm{CAD}$ diagnosis including myocardial infarction, acute coronary syndrome, chronic stable angina, or coronary stenosis of $>50 \%[33 \cdot 0]$. Accordingly, the term ischemic heart disease (IHD) is more appropriate for women than CHD. Abnormal coronary reactivity, microvascular dysfunction, and plaque erosion/distal microembolization contribute to a female-specific IHD pathophysiology. Using IHD, rather than obstructive CHD, covers the whole spectrum of the disease in women and could allow further identification of genes in studies focusing on women [15].

Another issue is whether studies have included prevalent or incident CHD cases. A recent GWAS was the first to investigate incident cases [ $80 \bullet$ ]. Importantly, a new gene was identified that was not identified in prior GWAS of prevalent cases while the 9p21 region and other genes identified in studies with prevalent cases were only marginally associated or not associated with incident disease. One explanation is that SNPs in the 9p21 region are associated with increased risk as well as survival after onset of CHD.

\section{Racial Disparities in Genetics of Coronary Heart Disease in Women}

The majority of genetic studies include only those of European ancestry. The differences among the various race/ ethnic groups in CHD morbidity and mortality are well documented [2]. The importance of sex and race/ethnicity in genetic studies of CVD has been recognized [81]. One GWAS of incident CHD was conducted in African Americans [82]. The 
discovery cohort included both women and men, but the replication cohort included only women. One SNP near PFTAIRE-1, which is involved in cell proliferation, was genome-wide significant and replicated. The region was not implicated in the incident CHD GWAS that pooled men and women $[80 \bullet \cdot$.

A recent study was designed to understand how to facilitate inclusion of minority individuals in genetic studies [83]. Starting with a large random sample from a community, they performed telephone screening interviews, determined eligibility, and then recruited participants for a genetic study on dependence on cigarettes and nicotine. In zip codes with a high proportion of African Americans, compared to those with very low proportions of African Americans, there was a significantly higher proportion of individuals with incorrect telephone numbers or addresses but a lower proportion of individuals who did not answer the telephone or refused the interview. Importantly, a significantly higher proportion of eligible African Americans participated in the genetic study compared to eligible European Americans (71 \% versus $54 \%$ ). Results suggest that increasing the number of African Americans in genetic studies and registries may be achieved by increasing efforts to locate and contact them. This study did not address some other issues regarding possible lack of participation of minorities in research studies because of mistrust or limited access to health care.

\section{Genetics of Women's Heart Disease: the Biological Basis for Precision Medicine}

Contrary to the traditional approach of "one size fits all," precision medicine aims to tailor disease prevention, treatment, and prognosis regimens [84•]. Sex-specific CHD research will lead to a new understanding of the pathophysiology of CHD in women, allowing for a comprehensive disease definition and classification. Taking into account individual variability in the role of genetic, environmental, and social factors as well as their interaction in the pathophysiology of atherosclerosis would serve to implement effective preventive or therapeutic interventions. As gene therapy becomes a promising new addition to conventional therapies, the sexspecific as well as the race/ethnic-specific genetic basis of the atherosclerosis assumes more significance.
Table 1 Accounting for sex differences in genetic studies of atherosclerosis: challenges and recommendations

\begin{tabular}{|c|c|}
\hline Challenges & Recommendations \\
\hline $\begin{array}{l}\text { Lack of meaningful comparisons between women } \\
\text { and men due to inadequate statistical power }\end{array}$ & Design sufficiently powered studies \\
\hline $\begin{array}{l}\text { Bias resulting from disproportionate blend of women } \\
\text { and men in case and control samples }\end{array}$ & $\begin{array}{l}\text { Careful consideration of the proportion of women and } \\
\text { men in case-control studies }\end{array}$ \\
\hline \multirow[t]{3}{*}{$\begin{array}{l}\text { Differential misclassification of cases and controls } \\
\text { between women and men due to suboptimal } \\
\text { accuracy in phenotyping (i.e., trait heterogeneity) }\end{array}$} & $\begin{array}{l}\text { Define the trait with sufficient specificity taking into } \\
\text { account sex differences in pathophysiology of } \\
\text { atherosclerosis }\end{array}$ \\
\hline & $\begin{array}{l}\text { Conduct sex-specific studies across the entire spectrum } \\
\text { of atherosclerosis and study the intermediate pheno- } \\
\text { types }\end{array}$ \\
\hline & $\begin{array}{l}\text { Distinguish prevalent versus incident traits in genetic } \\
\text { studies of atherosclerosis }\end{array}$ \\
\hline $\begin{array}{l}\text { Inconsistent results due to race/ethnic differences in } \\
\text { CHD morbidity and mortality }\end{array}$ & $\begin{array}{l}\text { Sufficient inclusion of individuals from different race/ } \\
\text { ethnic groups to investigate differences in genetic } \\
\text { associations with atherosclerosis }\end{array}$ \\
\hline \multirow[t]{4}{*}{$\begin{array}{l}\text { Spurious findings due to the interaction of genes with } \\
\text { other genetic and/or environmental factors }\end{array}$} & $\begin{array}{l}\text { Perform a systematic genetic assessment targeting the } \\
\text { true causal variants }\end{array}$ \\
\hline & Apply proper data analysis techniques \\
\hline & $\begin{array}{l}\text { Perform appropriate examination of subgroup } \\
\text { comparisons or interaction terms }\end{array}$ \\
\hline & $\begin{array}{l}\text { Consider possible sex hormone-mediated epigenetic } \\
\text { mechanisms }\end{array}$ \\
\hline \multirow{4}{*}{$\begin{array}{l}\text { Lack of comprehensive evidence supporting the } \\
\text { biological relevance of findings }\end{array}$} & Assess the internal and external validity of findings \\
\hline & Take forward the discovered genetic loci for replication \\
\hline & $\begin{array}{l}\text { Use available databases to identify function of genes } \\
\text { and relationship between genetic variation and gene } \\
\text { expression }\end{array}$ \\
\hline & $\begin{array}{l}\text { Undertake functional studies to understand the function } \\
\text { of the identified genetic variants }\end{array}$ \\
\hline
\end{tabular}




\section{Directions for Future Research}

CHD is increasingly recognized as a pathophysiological continuum [85], emphasizing the notion that intervention at any point along this path can modify disease progression. Disease progression does not occur as a sequence of discrete, tandem incidents but instead the phases of disease progression overlap. Therefore, sex-specific studies across the entire spectrum of atherosclerosis are needed. Studying intermediate phenotypes in the continuum, including CAC, CIMT, or endothelial function, may help disentangle sex differences in disease susceptibility and progression. Moreover, focusing on quantitative atherosclerosis markers might overcome some of the limitations regarding the heterogeneity in discrete CHD event ascertainment for women and men. In GWAS, the imprecision involved in measurement of quantitative phenotypes may not have large systematic effects on location of significant associations, but assessing repeatability of the phenotype is recommended [86].

The vast majority of identified SNPs do not result in protein changes. Instead, they could be, for example, long noncoding RNA, microRNA (small noncoding RNA that functions in RNA silencing and post-transcriptional regulation), or some transcribed or regulatory element. Thus, understanding the function of the identified SNPs is crucial for understanding their role in the disease process [87••]. One approach is to use available databases such as Encyclopedia of DNA elements (ENCODE) to identify the function of specific SNPs. Another is to utilize the data collected by the Genotype-Tissue Expression (GTEx) project, which provides a database and associated tissue bank to study the relationship between genetic variation and gene expression in human tissues [88]. A complete list of available databases is updated each year [89•]. Another approach is to study the function of a specific SNP and genomic region. For example, Yang and colleagues [90••] focused on an SNP in the COL4A2 gene that has been shown to be associated with CHD in GWAS. They studied the functional effect of this variant in primary cultures of vascular smooth cells and endothelial cells from individuals with different genotypes at this SNP. They found differences in gene expression levels of COL4A2 and COL4A1 (COL4A1 and $C O L 4 A 2$ reside next to each other and share common transcriptional regulatory sequences). Additional immunohistochemical and histological studies of ex vivo atherosclerotic coronary arteries identified plaque differences dependent on genotype. Together, these studies, as well as their research in patients with angiographically documented disease, provide a mechanistic explanation for the association between the genetic variants and CHD [90••]. One recent study used reporter gene assays, computational predictions, and epigenomic marks to assess activity of an enhancer region active in multiple human tissues [91] while another study used integrative genomic, epigenomic, and transcriptomic profiling of perturbed human coronary artery smooth muscle cells and tissues to begin to identify causal regulatory variation and mechanisms responsible for CHD associations [92•].

More recent studies are now examining exomes [93, 94••]. In addition, there are large-scale whole-genome sequencing projects in progress. The National Heart Lung and Blood Institute at the National Institutes of Health in the USA is sequencing more than 62,000 individuals from more than 30 studies, many with measures of CHD and risk factors through the Trans-Omics for Precision Medicine (TOPMed) Program (http://www.nhlbi.nih.gov/research/resources/nhlbiprecision-medicine-initiative/topmed). Hopefully, these various approaches will result in large samples of women for genomic studies of CHD.

\section{Limitations of Review}

There are many articles in the literature on the genetics of CHD and its risk factors that could not be included. We did not consider other manifestations of CVD that are relevant for women such as stroke, heart failure, and peripheral artery disease. We included only candidate gene and GWAS studies based on microarray data and did not include any GWAS based on exome sequencing or whole genome sequencing data that are just becoming available.

\section{Conclusions}

Sex-based differences in the heart, in vessel function, and in major manifestations of CVD have been recognized. The influence of sex on CHD is increasingly being explored at cellular, molecular, and genetic levels. Challenges and recommendations to account for sex differences in genetic studies of atherosclerosis are summarized in Table 1. So far, sexspecific genetic studies of atherosclerosis are sparse, mostly due to lack of power or lack of appreciation for sex differences. Sufficiently large hypothesis-free GWAS or candidate gene studies with a priori, clearly defined hypotheses are needed. Accurate phenotyping and inclusion of relevant outcomes in women, consideration of possible gene-gene and gene-environment interactions, and sex hormone-mediated epigenetic mechanisms are of importance. Finally, discovered genetic loci should be taken forward for replication and functional studies to elucidate plausible underlying biological mechanisms.

\section{Compliance with Ethical Standards}

Conflict of Interest Maryam Kavousi is supported by the VENI grant (91616079) from The Netherlands Organization for Health Research and Development (ZonMw).

Lawrence F. Bielak and Patricia A. Peyser declare that they have no conflict of interest. 
Human and Animal Rights and Informed Consent This article does not contain any studies with human or animal subjects performed by any of the authors.

Open Access This article is distributed under the terms of the Creative Commons Attribution 4.0 International License (http:// creativecommons.org/licenses/by/4.0/), which permits unrestricted use, distribution, and reproduction in any medium, provided you give appropriate credit to the original author(s) and the source, provide a link to the Creative Commons license, and indicate if changes were made.

\section{References}

Recently published papers of particular interest have been highlighted as:

- Of importance

•- Of major importance

1. Nichols M, Townsend N, Scarborough P, Rayner M. Cardiovascular disease in Europe: epidemiological update 2015. Eur Heart J. 2015;36:2950-9.

2. Mozaffarian D, Benjamin EJ, Go AS, Arnett DK, Blaha MJ, Cushman M, et al. Heart Disease and Stroke Statistics-2016 update: a report from the American Heart Association. Circulation. 2016;133:e38-60

3. Mosca L, Barrett-Connor E, Wenger NK. Sex/gender differences in cardiovascular disease prevention: what a difference a decade makes. Circulation. 2011;124:2145-54.

4. Hirsch AT, Allison MA, Gomes AS, Corriere MA, Duval S, Ershow AG, et al. A call to action: women and peripheral artery disease: a scientific statement from the American Heart Association. Circulation. 2012;125:1449-72.

5. Lloyd-Jones DM, Larson MG, Beiser A, Levy D. Lifetime risk of developing coronary heart disease. Lancet. 1999;353:89-92.

6. Lloyd-Jones DM, Larson MG, Leip EP, Beiser A, D'Agostino RB, Kannel WB, et al. Lifetime risk for developing congestive heart failure: the Framingham Heart Study. Circulation. 2002;106: 3068-72.

7. Seshadri S, Beiser A, Kelly-Hayes M, Kase CS, Au R, Kannel WB, et al. The lifetime risk of stroke: estimates from the Framingham Study. Stroke. 2006;37:345-50.

8. Mosca L, Benjamin EJ, Berra K, Bezanson JL, Dolor RJ, LloydJones DM, et al. Effectiveness-based guidelines for the prevention of cardiovascular disease in women - 2011 update: a guideline from the American Heart Association. Circulation. 2011;123: 1243-62.

9. Ford ES, Capewell S. Coronary heart disease mortality among young adults in the U.S. From 1980 through 2002. Concealed leveling of mortality rates. J Am Coll Cardiol. 2007;50:2128-32.

10. Wilmot KA, O'Flaherty M, Capewell S, Ford ES, Vaccarino V. Coronary heart disease mortality declines in the United States from 1979 through 2011: Evidence for stagnation in young adults, especially women. Circulation. 2015;132:997-1002. This study demonstrates important heterogeneity in CHD mortality trends.

11. Ni H, Coady S, Rosamond W, Folsom AR, Chambless L, Russell SD, et al. Trends from 1987 to 2004 in sudden death due to coronary heart disease: the Atherosclerosis Risk in Communities (ARIC) study. Am Heart J. 2009;157:46-52.

12. Singh RB, Mengi SA, Xu Y-J, Arneja AS, Dhalla NS. Pathogenesis of atherosclerosis: a multifactorial process. Exp Clin Cardiol. 2002;7:40-53.
13. Huxley R, Barzi F, Woodward M. Excess risk of fatal coronary heart disease associated with diabetes in men and women: metaanalysis of 37 prospective cohort studies. BMJ. 2006;332:73-8.

14. Huxley RR, Woodward M. Cigarette smoking as a risk factor for coronary heart disease in women compared with men: a systematic review and meta-analysis of prospective cohort studies. Lancet. 2011;378:1297-305.

15. Shaw LJ, Bugiardini R, Merz CNB. Women and ischemic heart disease. Evolving knowledge. J Am Coll Cardiol. 2009;54:156175. Elsevier Inc.

16. Quyyumi AA. Women and ischemic heart disease: pathophysiologic implications from the Women's Ischemia Syndrome Evaluation (WISE) Study and future research steps. J Am Coll Cardiol. 2006;47:S66-71.

17. Villar IC, Hobbs AJ, Ahluwalia A. Sex differences in vascular function: implication of endothelium-derived hyperpolarizing factor. J Endocrinol. 2008;197:447-62.

18. Song C, Chang Z, Magnusson PKE, Ingelsson E, Pedersen NL. Genetic factors may play a prominent role in the development of coronary heart disease dependent on important environmental factors. J Intern Med. 2014;275:631-9.

19. Kardia SLR, Modell SM, Peyser PA. Family-centered approaches to understanding and preventing coronary heart disease. Am J Prev Med. 2003;24:143-51.

20. Eichner JE, Dunn ST, Perveen G, Thompson DM, Stewart KE, Stroehla BC. Human Genome Epidemiology (HuGE) review. Am J Epidemiol. 2002;155:487-95.

21. Wilson PW, Myers RH, Larson MG, Ordovas JM, Wolf PA, Schaefer EJ. Apolipoprotein E alleles, dyslipidemia, and coronary heart disease. The Framingham Offspring Study. JAMA. 1994;272: 1666-71.

22. Ward H, Mitrou PN, Bowman R, Luben R, Wareham NJ, Khaw K$\mathrm{T}$, et al. Genotype, lipids, and coronary heart disease risk. Arch Intern Med. 2009;169:1424-9.

23. Silander K, Alanne M, Kristiansson K, Saarela O, Ripatti S, Auro $\mathrm{K}$, et al. Gender differences in genetic risk profiles for cardiovascular disease. PLoS One. 2008;3:e3615.

24. Silva MCP, Njajou OT, Alizadeh BZ, Hofman A, Witteman JCM, van Duijn $\mathrm{CM}$, et al. HFE gene mutations increase the risk of coronary heart disease in women. Eur J Epidemiol. 2010;25:643-9.

25. Tabor HK, Risch NJ, Myers RM. Candidate-gene approaches for studying complex genetic traits: practical considerations. Nat Rev Genet. 2002;3:391-7.

26. Auton A, Brooks LD, Durbin RM, Garrison EP, Kang HM, Korbel $\mathrm{JO}$, et al. A global reference for human genetic variation. Nature. 2015;526:68-74.

27.• Welter D, MacArthur J, Morales J, Burdett T, Hall P, Junkins H, et al. The NHGRI GWAS Catalog, a curated resource of SNP-trait associations. Nucleic Acids Res. 2014;42:D1001-6. Description of publically available and continuously updated collection of published GWAS that meet specific criteria.

28. Larson MG, Atwood LD, Benjamin EJ, Cupples LA, D'Agostino RBS, Fox CS, et al. Framingham Heart Study $100 \mathrm{~K}$ project: genome-wide associations for cardiovascular disease outcomes. BMC Med Genet. 2007;8 Suppl 1:S5.

29. Samani NJ, Erdmann J, Hall AS, Hengstenberg C, Mangino M, Mayer B, et al. Genomewide association analysis of coronary artery disease. N Engl J Med. 2007;357:443-53.

30. Helgadottir A, Thorleifsson G, Manolescu A, Gretarsdottir S, Blondal T, Jonasdottir A, et al. A common variant on chromosome 9p21 affects the risk of myocardial infarction. Science. 2007;316: 1491-3.

31. McPherson R, Pertsemlidis A, Kavaslar N, Stewart A, Roberts R, Cox DR, et al. A common allele on chromosome 9 associated with coronary heart disease. Science. 2007;316:1488-91. 
32. Genome-wide association study of 14,000 cases of seven common diseases and 3,000 shared controls. Nature. 2007;447:661-78

33.• Nikpay M, Goel A, Won H-H, Hall LM, Willenborg C, Kanoni S, et al. A comprehensive 1,000 Genomes-based genome-wide association meta-analysis of coronary artery disease. Nat Genet. 2015;47:1121-30. GWAS of $\sim \mathbf{1 8 5 , 0 0 0}$ CHD cases and controls identifying 10 new loci.

34.• McPherson R, Tybjaerg-Hansen A. Genetics of coronary artery disease. Circ Res. 2016;118:564-78. Recent review of evidence for role of genes in CHD, approaches to understand function of identified genes, and potential clinical utility.

35. Orozco G, Ioannidis JPA, Morris A, Zeggini E. Sex-specific differences in effect size estimates at established complex trait loci. Int $\mathrm{J}$ Epidemiol. 2012;41:1376-82.

36. Goodarzynejad H, Boroumand M, Behmanesh M, Ziaee S, Jalali A. The rs5888 single nucleotide polymorphism in scavenger receptor class B type 1 (SCARB1) gene and the risk of premature coronary artery disease: a case-control study. Lipids Health Dis. 2016;15:7. Study presenting a sex-dependent association between a SNP in SCARB1 and premature disease.

37.• Hartiala JA, Tang WHW, Wang Z, Crow AL, Stewart AFR, Roberts $\mathrm{R}$, et al. Genome-wide association study and targeted metabolomics identifies sex-specific association of CPS1 with coronary artery disease. Nat Commun. 2016;7:10558. Study shows genes involved in glycine metabolism and/or the urea cycle could be novel sexspecific genes for atherosclerosis.

38. Schunkert H, Konig IR, Kathiresan S, Reilly MP, Assimes TL, Holm $\mathrm{H}$, et al. Large-scale association analysis identifies 13 new susceptibility loci for coronary artery disease. Nat Genet. 2011;43:333-8.

39. Wise AL, Gyi L, Manolio TA. eXclusion: toward integrating the $\mathrm{X}$ chromosome in genome-wide association analyses. Am J Hum Genet The American Society of Human Genetics. 2013;92:643-7.

40. Weng L, Taylor KD, Chen Y-DI, Sopko G, Kelsey SF, Bairey Merz $\mathrm{CN}$, et al. Genetic loci associated with nonobstructive coronary artery disease in Caucasian women. Physiol Genomics. 2016;48:12-20.

41. Voight BF, Kang HM, Ding J, Palmer CD, Sidore C, Chines PS, et al. The metabochip, a custom genotyping array for genetic studies of metabolic, cardiovascular, and anthropometric traits. PLoS Genet. 2012;8:e1002793.

42. Brummett BH, Babyak MA, Jiang R, Shah SH, Becker RC, Haynes $\mathrm{C}$, et al. A functional polymorphism in the 5HTR2C gene associated with stress responses also predicts incident cardiovascular events. PLoS One. 2013;8:e82781.

43. Vitale C, Mendelsohn ME, Rosano GMC. Gender differences in the cardiovascular effect of sex hormones. Nat Rev Cardiol. 2009;6: $532-42$.

44. Waddington $\mathrm{CH}$. Organisers and Genes. Cambridge [Eng]: University Press; 1940.

45. Sadakierska-Chudy A, Filip M. A comprehensive view of the epigenetic landscape. Part II: histone post-translational modification, nucleosome level, and chromatin regulation by ncRNAs. Neurotox Res. 2015;27:172-97.

46. Jaenisch R, Bird A. Epigenetic regulation of gene expression: how the genome integrates intrinsic and environmental signals. Nat Genet. 2003;33(Suppl):245-54.

47. Ordovas JM, Smith CE. Epigenetics and cardiovascular disease. Nat Rev Cardiol. 2010;7:510-9. Review that focuses on epigenetic factors and their role in CVD risk, potential therapeutic agents, and nutritional compounds in prevention or treatment of disease.

48. Kaminsky Z, Wang S-C, Petronis A. Complex disease, gender and epigenetics. Ann Med Sweden. 2006;38:530-44.

49. Kardys I, Vliegenthart R, Oudkerk M, Hofman A, Witteman JCM. The female advantage in cardiovascular disease: do vascular beds contribute equally? Am J Epidemiol. 2007;166:403-12.
50. Allison MA, Budoff MJ, Nasir K, Wong ND, Detrano R, Kronmal $\mathrm{R}$, et al. Ethnic-specific risks for atherosclerotic calcification of the thoracic and abdominal aorta (from the Multi-Ethnic Study of Atherosclerosis). Am J Cardiol. 2009;104:812-7.

51. Nasir K, Shaw LJ, Liu ST, Weinstein SR, Mosler TR, Flores PR, et al. Ethnic differences in the prognostic value of coronary artery calcification for all-cause mortality. J Am Coll Cardiol. 2007;50: 953-60.

52. Rumberger JA. The ethnic Rosetta stone: translating risk factors, plaque scores, and mortality. J. Am. Coll. Cardiol. 2007. p. 961-3

53. Alexopoulos N, Raggi P. Calcification in atherosclerosis. Nat Rev Cardiol. 2009;6:681-8.

54. Peyser P, Bielak L, Chu JS, Turner ST, Ellsworth DL, Boerwinkle E, et al. Heritability of coronary artery calcium quantity measured by electron beam computed tomography in asymptomatic adults. Circulation. 2002;106:304-8.

55. O'Donnell CJ, Kavousi M, Smith AV, Kardia SLR, Feitosa MF, Hwang S-JJ, et al. Genome-wide association study for coronary artery calcification with follow-up in myocardial infarction. Circulation. 2011;124:2855-64. First large-scale GWAS on $\mathrm{CAC}$ that identified two regions in the genome associated with CAC.

56. Wojczynski MK, Li M, Bielak LF, Kerr KF, Reiner AP, Wong ND, et al. Genetics of coronary artery calcification among African Americans, a meta-analysis. BMC Med Genet. 2013;14:75.

57. Ellsworth DL, Bielak LF, Turner ST, Sheedy 2nd PS, Boerwinkle E, Peyser PA. Gender- and age-dependent relationships between the E-selectin S128R polymorphism and coronary artery calcification. J Mol Med. 2001;79:390-8.

58. Iovannisci DM, Lammer EJ, Steiner L, Cheng S, Mahoney LT, Davis $\mathrm{PH}$, et al. Association between a leukotriene C4 synthase gene promoter polymorphism and coronary artery calcium in young women: the Muscatine Study. Arterioscler Thromb Vasc Biol. 2007:27:394-9.

59. Sinning C, Wild PS, Echevarria FMO, Wilde S, Schnabel R, Lubos E, et al. Sex differences in early carotid atherosclerosis (from the community-based Gutenberg-Heart Study). Am J Cardiol. 2011;107:1841-7.

60. Lin H-F, Huang L-C, Chen C-H, Hsu CY, Lin R-T, Juo S-HH. Age and sex differences in the effect of parental stroke on the progression of carotid intima-media thickness. Atherosclerosis. 2015;241: 229-33.

61. Zhao J, Cheema FA, Bremner JD, Goldberg J, Su S, Snieder H, et al. Heritability of carotid intima-media thickness: a twin study. Atherosclerosis. 2008;197:814-20.

62. Bis JC, Kavousi M, Franceschini N, Isaacs A, Abecasis GR, Schminke U, et al. Meta-analysis of genome-wide association studies from the CHARGE consortium identifies common variants associated with carotid intima media thickness and plaque. Nat Genet. 2011;43:940-7. First large-scale GWAS on CIMT identified three regions in the genome associated with IMT.

63. Dong C, Della-Morte D, Beecham A, Wang L, Cabral D, Blanton $\mathrm{SH}$, et al. Genetic variants in LEKR1 and GALNT10 modulate sexdifference in carotid intima-media thickness: a genome-wide interaction study. Atherosclerosis. 2015;240:462-7. GWAS of CIMT that identified two genes that modify association between sex and CIMT.

64. Gertow K, Sennblad B, Strawbridge RJ, Ohrvik J, Zabaneh D, Shah $\mathrm{S}$, et al. Identification of the BCAR1-CFDP1-TMEM170A locus as a determinant of carotid intima-media thickness and coronary artery disease risk. Circ Cardiovasc Genet. 2012;5:656-65.

65. Boardman-Pretty F, Smith AJP, Cooper J, Palmen J, Folkersen L, Hamsten A, et al. Functional analysis of a carotid intima-media thickness locus implicates BCAR1 and suggests a causal variant. Circ Cardiovasc Genet. 2015;8:696-706. This study identified an SNP associated with slower CIMT progression in women, but 
not men, and explored the function using bioinformatics and in vitro functional assays.

66. Cohn JN, Quyyumi AA, Hollenberg NK, Jamerson KA. Surrogate markers for cardiovascular disease: functional markers. Circulation. 2004;109:IV31-46.

67. Deanfield JE, Halcox JP, Rabelink TJ. Endothelial function and dysfunction: testing and clinical relevance. Circulation. 2007;115: 1285-95.

68. Wang J, Bingaman S, Huxley VH. Intrinsic sex-specific differences in microvascular endothelial cell phosphodiesterases. Am J Physiol Heart Circ Physiol. 2010;298:H1146-54.

69. Brar V, Gill S, Cardillo C, Tesauro M, Panza JA, Campia U. Sexspecific effects of cardiovascular risk factors on endotheliumdependent dilation and endothelin activity in middle-aged women and men. PLoS One. 2015;10:e0121810.

70. Zhao J, Cheema FA, Reddy U, Bremner JD, Su S, Goldberg J, et al. Heritability of flow-mediated dilation: a twin study. J Thromb Haemost. 2007;5:2386-92.

71. Vasan RS, Larson MG, Aragam J, Wang TJ, Mitchell GF, Kathiresan S, et al. Genome-wide association of echocardiographic dimensions, brachial artery endothelial function and treadmill exercise responses in the Framingham Heart Study. BMC Med Genet. 2007;8 Suppl 1:S2.

72. Yoshino S, Cilluffo R, Prasad M, Best PJM, Atkinson EJ, Aoki T, et al. Sex-specific genetic variants are associated with coronary endothelial dysfunction. J Am Heart Assoc. 2016;5:e02544.

73. Gustavsson J, Mehlig K, Leander K, Strandhagen E, Bjorck L, Thelle DS, et al. Interaction of apolipoprotein E genotype with smoking and physical inactivity on coronary heart disease risk in men and women. Atherosclerosis. 2012;220:486-92.

74. Patsopoulos NA, Tatsioni A, Ioannidis JPA. Claims of sex differences: an empirical assessment in genetic associations. JAMA. 2007;298:880-93. Review of specific criteria needed in a study to declare differences in genetic effects between women and men.

75. IOM (Institute of Medicine). Sex-specific reporting of scientific research: a workshop summary. Washington: The National Academies Press; 2012.

76. Hingorani ADA, van der Windt DA, Riley RD, Abrams K, Moons KGM, Steyerberg EW, et al. Prognosis research strategy (PROGRESS) 4: stratified medicine research. BMJ. 2013;5793:1-9.

77. Kavousi M. Tools and techniques - statistical. Confounding and effect measure modification: analysing sex in cardiovascular research. EuroIntervention. 2016;12:404-7.

78. Thornton-Wells TA, Moore JH, Haines JL. Dissecting trait heterogeneity: a comparison of three clustering methods applied to genotypic data. BMC Bioinformatics. 2006;7:204.

79. Bairey Merz CN, Shaw LJ, Reis SE, Bittner V, Kelsey SF, Olson M, et al. Insights from the NHLBI-Sponsored Women's Ischemia Syndrome Evaluation (WISE) Study: part II: gender differences in presentation, diagnosis, and outcome with regard to gender-based pathophysiology of atherosclerosis and macrovascular and microvascular coronary disease. J Am Coll Cardiol. 2006;47:S21-9.

80.• Dehghan A, Bis JC, White CC, Smith AV, Morrison AC, Cupples LA, et al. Genome-wide association study for incident myocardial infarction and coronary heart disease in prospective cohort studies: the CHARGE Consortium. PLoS One. 2016;11:e0144997. Largescale GWAS on incident myocardial infarction and CHD identified novel locus and highlighted potential genetic differences between incident and prevalent disease.

81. Winham SJ, de Andrade M, Miller VM. Genetics of cardiovascular disease: importance of sex and ethnicity. Atherosclerosis Elsevier Ltd. 2015;241:219-28.
82. Barbalic M, Reiner AP, Wu C, Hixson JE, Franceschini N, Eaton $\mathrm{CB}$, et al. Genome-wide association analysis of incident coronary heart disease (CHD) in African Americans: A short report. PLoS Genet. 2011;7:e1002199.

83. Hartz SM, Johnson EO, Saccone NL, Hatsukami D, Breslau N, Bierut LJ. Inclusion of African Americans in genetic studies: what is the barrier? Am J Epidemiol. 2011;174:336-44.

84. Antman EM, Loscalzo J. Precision medicine in cardiology. Nat. Rev. Cardiol. 2016; doi:10.1038/nrcardio.2016.101. [Epub ahead of print]. Review discusses advances in biomedicine and the opportunities to improve cardiovascular health.

85. Dzau VJ, Antman EM, Black HR, Hayes DL, Manson JE, Plutzky J, et al. The cardiovascular disease continuum validated: clinical evidence of improved patient outcomes: part I: pathophysiology and clinical trial evidence (risk factors through stable coronary artery disease). Circulation. 2006;114:2850-70.

86. Barendse W. The effect of measurement error of phenotypes on genome wide association studies. BMC Genomics. 2011;12:232.

87.• Nurnberg ST, Zhang H, Hand NJ, Bauer RC, Saleheen D, Reilly MP, et al. From loci to biology: functional genomics of genomewide association for coronary disease. Circ Res. 2016;118:586606. Review of tools used in functional genomics to address questions once a SNP or locus has been associated with disease.

88. The Genotype-Tissue Expression (GTEx) project. Nat. Genet. 2013:45:580-5

89. Rigden DJ, Fernandez-Suarez XM, Galperin MY. The 2016 database issue of Nucleic Acids Research and an updated molecular biology database collection. Nucleic Acids Res. 2016;44:D1-6. Provides extensive information on a vast number of publically available molecular biology databases that is updated each year.

90.• Yang W, Ng FL, Chan K, Pu X, Poston RN, Ren M, et al. Coronaryheart-disease-associated genetic variant at the COL4A1/COL4A2 locus affects COL4A1/COL4A2 expression, vascular cell survival, atherosclerotic plaque stability and risk of myocardial infarction. PLoS Genet. 2016;12(Yang W, Ng FL, Chan K, Pu X, Poston RN, Ren M):e1006127. Example of studies of gene expression, smooth muscle cells, and plaque stability to understand the mechanisms for a SNP associated with $\mathrm{CHD}$.

91. Smith JG, Felix JF, Morrison AC, Kalogeropoulos A, Trompet S, Wilk JB, et al. Discovery of genetic variation on chromosome $5 \mathrm{q} 22$ associated with mortality in heart failure. PLoS Genet. 2016;12: e1006034

92. Miller CL, Pjanic M, Wang T, Nguyen T, Cohain A, Lee JD, et al. Integrative functional genomics identifies regulatory mechanisms at coronary artery disease loci. Nat Commun. 2016;7:12092. Study uses integrative genomic, epigenomic, and transcriptomic profiling of perturbed human coronary artery smooth muscle cells and tissues to identify causal mechanisms for $\mathrm{CHD}$.

93. Yu B, Li AH, Muzny D, Veeraraghavan N, de Vries PS, Bis JC, et al. Association of rare loss-of-function alleles in HAL, serum histidine: levels and incident coronary heart disease. Circ Cardiovasc Genet. 2015;8:351-5.

94.• Zanoni P, Khetarpal SA, Larach DB, Hancock-Cerutti WF, Millar JS, Cuchel M, et al. Rare variant in scavenger receptor BI raises HDL cholesterol and increases risk of coronary heart disease. Science. 2016;351:1166-71. Study using targeted sequencing of coding regions of $\sim 990$ lipid modifying genes and identified a variant associated with high HDL-C and increased risk of CHD; the variant impairs posttranslational processing in both a human homozygote and in mice. 